\title{
A prognostic nomogram for the cancer- specific survival of patients with upper-tract urothelial carcinoma based on the Surveillance, Epidemiology, and End Results Database
}

\author{
Chengzhuo $\mathrm{Li}^{1,2+}$, Jin Yang ${ }^{1,2+}$, Fengshuo Xu ${ }^{1,2}$, Didi Han ${ }^{1,2}$, Shuai Zheng ${ }^{1,3}$, Rahel Elishilia Kaaya ${ }^{1,2}$,
} Shengpeng Wang ${ }^{4,5^{*}}$ and Jun Lyu ${ }^{1,2^{*}}$ (D)

\begin{abstract}
Background: The aim of this study was to establish a comprehensive nomogram for the cancer-specific survival (CSS) of patients with upper-tract urothelial carcinoma (UTUC) and compare it with the traditional American Joint Committee on Cancer (AJCC) staging system in order to determine its reliability.

Methods: This study analyzed 9505 patients with UTUC in the Surveillance, Epidemiology, and End Results (SEER) database. R software was used to randomly divided the patients in a 7-to-3 ratio to form a training cohort ( $n=$ 6653) and a validation cohort $(n=2852)$. Multivariable Cox regression was used to identify predictive variables. The new survival model was compared with the AJCC prognosis model using the concordance index (C-index), the area under the time-dependent receiver operating characteristics curve (AUC), the net reclassification improvement (NRI), the integrated discrimination improvement (IDI), calibration plotting, and decision-curve analysis (DCA).

Results: We have established a nomogram for determining the 3-, 5-, and 8-year CSS probabilities of UTUC patients. The nomogram indicates that the AJCC stage has the greatest influence on CSS in UTUC, followed by the age at diagnosis, surgery status, tumor size, radiotherapy status, histological grade, marital status, chemotherapy status, race, and finally sex. The C-index was higher for the nomogram than the AJCC staging system in both the training cohort ( 0.785 versus 0.747 ) and the validation cohort ( 0.779 versus 0.739 ). Calibration plotting demonstrated that the model has good calibration ability. The AUC, NRI, IDI, and DCA of the nomogram showed that it performs better than the AJCC staging system alone.
\end{abstract}

(Continued on next page)

\footnotetext{
* Correspondence: shengpeng.wang@xjtu.edu.cn; lyujun2020@jnu.edu.cn

${ }^{\dagger}$ Chengzhuo Li and Jin Yang contributed equally to this work.

${ }^{4}$ Cardiovascular Research Center, School of Basic Medical Sciences, Xi'an

Jiaotong University Health Science Center, Xi'an 710061, People's Republic of

China

'Department of Clinical Research, The First Affiliated Hospital of Jinan

University, Guangzhou, Guangdong, China

Full list of author information is available at the end of the article
}

C C The Author(s). 2020 Open Access This article is licensed under a Creative Commons Attribution 4.0 International License, which permits use, sharing, adaptation, distribution and reproduction in any medium or format, as long as you give appropriate credit to the original author(s) and the source, provide a link to the Creative Commons licence, and indicate if changes were made. The images or other third party material in this article are included in the article's Creative Commons licence, unless indicated otherwise in a credit line to the material. If material is not included in the article's Creative Commons licence and your intended use is not permitted by statutory regulation or exceeds the permitted use, you will need to obtain permission directly from the copyright holder. To view a copy of this licence, visit http://creativecommons.org/licenses/by/4.0/ The Creative Commons Public Domain Dedication waiver (http://creativecommons.org/publicdomain/zero/1.0/) applies to the data made available in this article, unless otherwise stated in a credit line to the data. 
(Continued from previous page)

Conclusions: This study is the first to establish a comprehensive UTUC nomogram based on the SEER database and evaluate it using a series of indicators. Our novel nomogram can help clinical staff to predict the 3-, 5-, and 8year CSS probabilities of UTUC patients more accurately than using the AJCC staging system.

Keywords: Upper-tract urothelial carcinoma, Nomogram, SEER, Cancer-specific survival

\section{Background}

Urothelial carcinoma is a type of urinary tumor that can occur in the upper urinary tract (renal pelvis and ureter) or the lower urinary tract (bladder and urethra). Although urothelial carcinoma is the fourth most common type of tumor [1], upper-tract urothelial carcinoma (UTUC) is a rare malignancy of the urinary system that accounts for about $10 \%$ of all renal tumors and $5 \%$ of all urothelial tumors [2]. UTUC includes carcinoma of the renal pelvis and ureter, and ureteral tumors are less common than renal pelvis tumors [3]. Most of the few studies that have investigated UTUC have combined UTUC with kidney cancer. However, since the incidence and mortality rates of UTUC have increased in recent years [3-5], the present study focused on analyzing UTUC alone.

Age at diagnosis and being male are known risk factors for UTUC [3]. Surgery is the preferred approach for treating UTUC, and nephroureterectomy with bladder cuff excision has been the mainstay treatment [6]. The roles of chemotherapy and radiotherapy in advanced disease have not been clearly demonstrated, but some studies have found chemotherapy to be beneficial [7]. UTUC has a more-aggressive clinical course and a worse prognosis than bladder cancer [8], and the currently available prognostic models of UTUC are inadequate.

The traditional American Joint Committee on Cancer (AJCC) staging system provides clinically significant prognoses of UTUC and is currently the main reference standard for the prognosis of clinical treatment [9]. However, the AJCC staging system does not incorporate the entire pathological nature of the tumor, excluding potentially important factors when predicting the prognosis such as demographic characteristics, tumor size, tumor location, and the treatment applied [10-12]. A nomogram is based on a prognostic model and it can clearly and concisely show how various prognostic factors influence certain outcome variables. A nomogram can be used to calculate the survival probability of individual patients, making it of great value in clinical practice [13].

The Surveillance, Epidemiology, and End Results (SEER) database has not previously been used to construct a prognostic nomogram for UTUC. Therefore, the purpose of this study was to establish a comprehensive nomogram that includes both demographic factors and clinicopathological features. The new prediction model was compared with the traditional AJCC staging system in order to determine its reliability. The developed nomogram has considerable clinical value in helping clinical staff to predict the 3-, 5-, and 8-year cancerspecific survival (CSS) probability of UTUC patients more comprehensively and on an individual basis.

\section{Methods \\ Source of data}

We analyzed data obtained from the SEER database. Part of that database is open to the public, and we also searched for additional chemotherapy data using the SEER*Stat software [14, 15]. We extracted UTUC patients from the SEER database in the following ways: [16] The primary sites of UTUC were selected using the codes "C65.9-Renal pelvis" and "C66.9-Ureter." All of the ICD-O-3 histology and behavior codes related to UTUC were included. Age at diagnosis, race, sex, and marital status were selected as demographic characteristics. The following pathological features were also included: primary site, histological grade, AJCC stage, tumor size, surgery status, radiotherapy status, and chemotherapy status. It is worth noting that the tumor histological grade is divided into four levels in the SEER database. The four-grade system describes the tumor as Grade I: well-differentiated; Grade II: moderately differentiated; Grade III: poorly differentiated; Grade IV: undifferentiated or anaplastic. We chose the AJCC stage based on the sixth edition of the Derived AJCC Stage Group. The tumor size was divided into three categories based on the diameter: $<2,2-4$, and $>4 \mathrm{~cm}[1,17]$. We classified the surgery status based on the records in the SEER database. "Yes" means surgery performed. "No" means three situations: patient died prior to recommended surgery, not recommended, and recommended but patient refused. The outcome in the study was death due to UTUC.

\section{Criteria for data selection}

This retrospective study initially identified 11,607 UTUC patients enrolled in the SEER database between 2004 and 2016 by applying the above criteria. However, 2073 patients were not included in the analysis due to the 
tumor size being unknown, with a further 29 patients rejected due to unclear histological tumor grading. Thus, we finally selected 9505 UTUC patients, and classified $70 \%(n=6653)$ of them into the training cohort for constructing the prognostic nomogram and $30 \%(n=2852)$ of them into the validation cohort for evaluating the constructed nomogram. The data screening process is shown in Fig. 1.

\section{Statistical analysis}

We performed a descriptive analysis of all of the abovementioned factors. The age at diagnosis was expressed as median and interquartile-range values, while other categorical variables were represented as percentages. Cox regression was used to screen for correlation factors for which $p=0.1$. We then established a nomogram that predicted the 3-, 5-, and 8-year CSS probabilities of UTUC.

After establishing the nomogram, we used a series of indicators to evaluate it. We first used the concordance index (C-index) and the area under the time-dependent receiver operating characteristics (ROC) curve (AUC) to evaluate the differentiation ability of the new model, and then supplemented this by adopting two relatively new indicators (net reclassification improvement [NRI] and integrated discrimination improvement [IDI]) to increase the accuracy and comprehensiveness of the comparisons
$[18,19]$. The consistency of survival probabilities predicted using the nomogram with the actual situation was evaluated by drawing calibration plots [20]. Finally, we used decision-curve analysis (DCA) to evaluate the clinical validity of the model [21].

All of the statistical analyses were performed using IBM SPSS Statistics software (version 23.0, SPSS, Chicago, IL, USA) and R software (version 3.4.1; http:// www.Rproject.org). $\mathrm{R}$ software was used to randomly divide the 9505 patients in a 7-to-3 ratio to the 2 study cohorts, and the log-rank test was also used to check that there were no significant differences between the cohorts. A bilateral probability value of $p<0.05$ was considered indicative of statistical significance.

It is not necessary to obtain informed patient consents for data obtained from the SEER database since it does not include information that can be used to identify individual patients.

\section{Results}

\section{Characteristics of the included patients}

The median age at diagnosis was 73 years (interquartile range, 65-80 years) in the training and validation cohorts. Most of the patients in the training and validation cohorts were male (59.2 and $60.0 \%$, respectively), white (87.7 and $87.3 \%)$, and married (87.5 and $87.9 \%)$. Among the tumor-related features, the primary site was

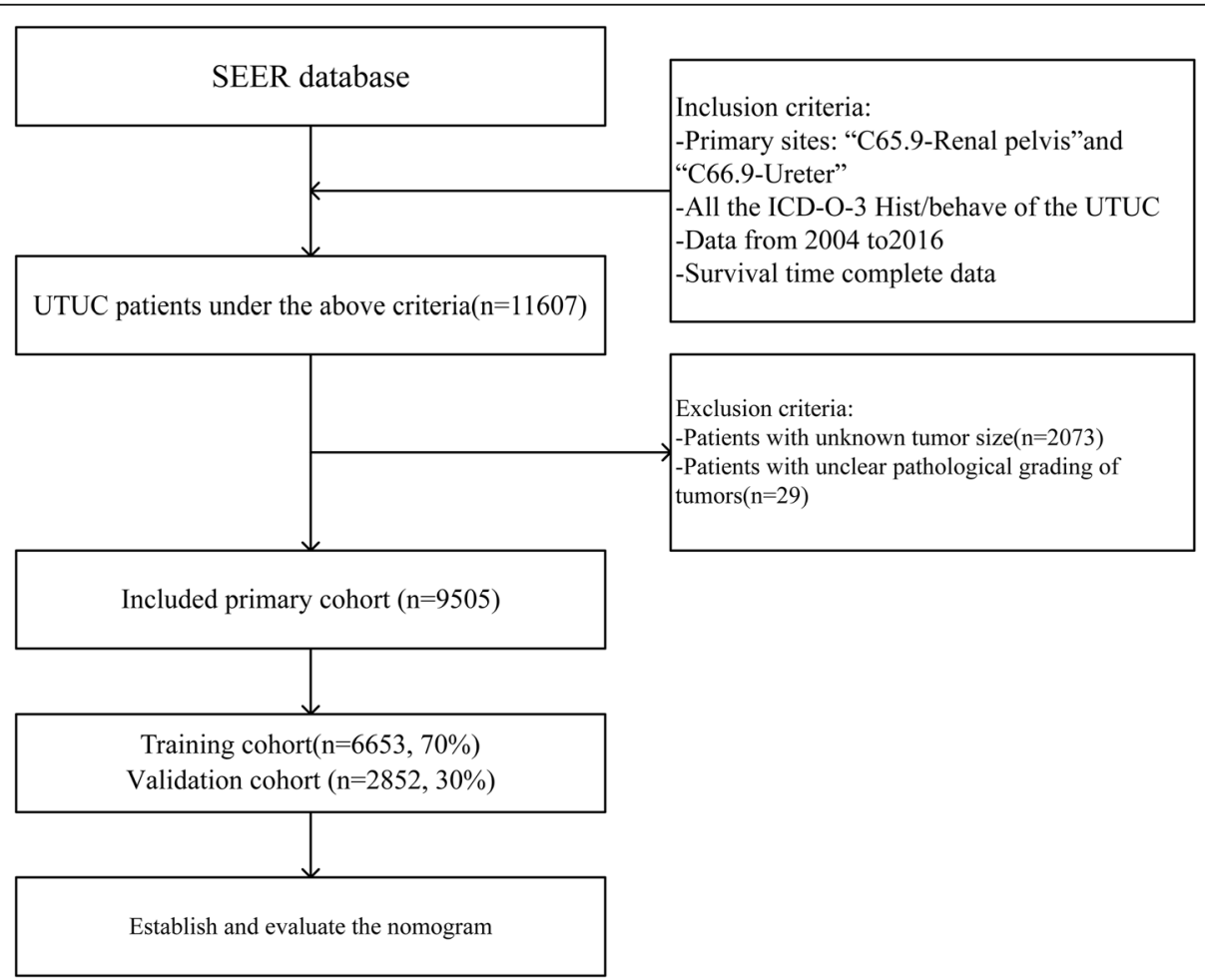

Fig. 1 Research flowchart 
predominantly in the renal pelvis $(65.7$ and $66.6 \%$ in the training and validation cohorts, respectively), with the remainder in the ureter. Most of the tumors were at histological grade IV and larger than $4 \mathrm{~cm}$ in both cohorts. The distribution of the different AJCC stages was close to uniform. Most of the patients had received surgery, with only a few receiving radiotherapy or chemotherapy in both cohorts. Table 1 summarizes the demographic and tumor characteristics of the two cohorts.

\section{Variable screening and nomogram establishment}

The age at diagnosis, sex, race, marital status, primary site, histological grade, tumor size, AJCC stages, surgery status, radiotherapy status, and chemotherapy status were entered into the multivariable Cox regression analysis. The results showed that all of the factors except the primary site were suitable for including in the model. The multivariable analysis revealed that the following factors were statistically significant: age at diagnosis (hazard ratio $[\mathrm{HR}]=1.016, p<0.001)$, female $(\mathrm{HR}=$ $1.144, p<0.01$ versus male), black $(\mathrm{HR}=1.223, p=0.088$ versus white), other race ( $\mathrm{HR}=1.186, p<0.05$ versus white), unmarried ( $\mathrm{HR}=1.236, p<0.05$ versus married), histological grade III ( $\mathrm{HR}=1.661, p<0.01$ versus grade I), histological grade IV $(\mathrm{HR}=1.791, p<0.001$ versus grade I), size $=2-4 \mathrm{~cm}(\mathrm{HR}=1.314, p<0.01$ versus size $<2 \mathrm{~cm})$, size $>4 \mathrm{~cm}(\mathrm{HR}=1.831, p<0.001$ versus size $<$ $2 \mathrm{~cm}$ ), AJCC stage II (HR $=1.609, p<0.001$ versus stage I), AJCC stage III (HR $=2.881, p<0.001$ versus stage I), AJCC stage IV ( $\mathrm{HR}=8.674, p<0.001$ versus stage I), no/ unknown surgery status $(\mathrm{HR}=2.936, p<0.001$ versus surgery), no/unknown radiotherapy status $(\mathrm{HR}=0.715$, $p<0.001$ versus radiotherapy), and no/unknown chemotherapy status $(\mathrm{HR}=1.254, p<0.001$ versus chemotherapy). Table 2 lists the results of the multivariable Cox regression analysis.

\section{Nomogram comparison and evaluation}

Figure 2 shows the nomogram for predicting the 3-, 5-, and 8-year CSS probabilities for UTUC patients that we established based on the findings of the multivariable Cox regression analysis. It can be seen from the nomogram that the AJCC stage has the greatest influence on the CSS probability for UTUC, followed by age at diagnosis, surgery status, tumor size, radiotherapy status, histological grade, marital status, chemotherapy status, race, and finally sex.

After establishing the nomogram we used a series of indicators to evaluate the performance of the new prediction model underpinning this nomogram. We first used the C-index to evaluate the effect of the nomogram, and found that this was higher for the nomogram than for the AJCC staging system in both the training
Table 1 Socio-demographic and clinical characteristics of patients in the study

\begin{tabular}{|c|c|c|c|}
\hline Variable & Training Cohort & Validation Cohort & $P$-value \\
\hline Number of Patients n (\%) & $6653(70)$ & $2852(30)$ & \\
\hline Age of diagnosis & $73(65-80)$ & $73(65-80)$ & 0.891 \\
\hline Sex n (\%) & & & 0.457 \\
\hline Male & $3937(59.2)$ & $1711(60.0)$ & \\
\hline Female & $2716(40.8)$ & $1141(40.0)$ & \\
\hline Race n (\%) & & & 0.442 \\
\hline White & $5832(87.7)$ & $2489(87.3)$ & \\
\hline Black & $279(4.2)$ & $136(4.8)$ & \\
\hline Other & $542(8.1)$ & $227(8.0)$ & \\
\hline Marital status n (\%) & & & 0.844 \\
\hline Married & $5820(87.5)$ & $2507(87.9)$ & \\
\hline Unmarried & $577(8.7)$ & $238(8.3)$ & \\
\hline Other & $256(3.8)$ & $107(3.8)$ & \\
\hline Site $n(\%)$ & & & 0.412 \\
\hline Renal pelvis & $4372(65.7)$ & 1899 (66.6) & \\
\hline Ureter & $2281(34.3)$ & $953(33.4)$ & \\
\hline Grade n (\%) & & & 0.538 \\
\hline I & $287(4.3)$ & $126(4.4)$ & \\
\hline$\|$ & $989(14.9)$ & $406(14.2)$ & \\
\hline III & $1990(29.9)$ & $850(29.8)$ & \\
\hline IV & $3387(50.9)$ & $1470(51.5)$ & \\
\hline Size n (\%) & & & 0.003 \\
\hline$<2$ & $1042(15.7)$ & $413(14.5)$ & \\
\hline$[2,4)$ & $2608(39.2)$ & $1053(36.9)$ & \\
\hline$\geq 4$ & $3003(45.1)$ & $1386(48.6)$ & \\
\hline AJCC stage $n(\%)$ & & & 0.901 \\
\hline । & $1900(28.6)$ & $836(29.3)$ & \\
\hline$\|$ & $1104(16.6)$ & $462(16.2)$ & \\
\hline III & 2125 (31.9) & $882(30.9)$ & \\
\hline IV & $1524(22.9)$ & $672(23.6)$ & \\
\hline Surgery n (\%) & & & 0.075 \\
\hline Yes & 6359 (95.6) & $2702(94.7)$ & \\
\hline NO/Unknown & $294(4.4)$ & $150(5.3)$ & \\
\hline Radiotherapy n (\%) & & & 0.942 \\
\hline Yes & $357(5.4)$ & $152(5.3)$ & \\
\hline NO/Unknown & $6296(94.6)$ & $2700(94.7)$ & \\
\hline Chemotherapy n (\%) & & & 0.217 \\
\hline Yes & $1344(20.2)$ & $608(21.3)$ & \\
\hline NO/Unknown & $5309(79.8)$ & $2244(78.7)$ & \\
\hline
\end{tabular}

cohort $(0.785$ versus 0.747$)$ and the validation cohort (0.779 versus 0.739$)$. We further compared ROC curves, which revealed that in the training cohort the 3-, 5-, and 8 -year AUC values were $0.832,0.825$, and 0.809 , 
Table 2 Selected variables by multivariable Cox regression analysis

\begin{tabular}{|c|c|c|c|}
\hline \multirow[t]{2}{*}{ Variable } & \multicolumn{3}{|c|}{ Multivariable analysis } \\
\hline & $\overline{H R^{a}}$ & $95 \% \mathrm{Cl}^{\mathrm{b}}$ & $P$-value \\
\hline Age of diagnosis & 1.016 & $1.011-1.021$ & $0.000^{* * *}$ \\
\hline \multicolumn{4}{|l|}{ Sex } \\
\hline Male & Reference & & \\
\hline Female & 1.144 & $1.039-1.260$ & $0.006^{* *}$ \\
\hline \multicolumn{4}{|l|}{ Race } \\
\hline White & Reference & & \\
\hline Black & 1.223 & $0.970-1.542$ & 0.088 \\
\hline Other & 1.186 & $1.014-1.388$ & $0.033^{*}$ \\
\hline \multicolumn{4}{|l|}{ Marital status } \\
\hline Married & Reference & & \\
\hline Unmarried & 1.236 & $1.048-1.458$ & $0.012^{*}$ \\
\hline Other & 0.907 & $0.691-1.190$ & 0.480 \\
\hline \multicolumn{4}{|l|}{ Site } \\
\hline Renal pelvis & Reference & & \\
\hline Ureter & 1.073 & $0.963-1.195$ & 0.200 \\
\hline \multicolumn{4}{|l|}{ Grade } \\
\hline । & Reference & & \\
\hline$\|$ & 1.029 & $0.709-1.494$ & 0.879 \\
\hline III & 1.661 & $1.175-2.346$ & $0.004^{* *}$ \\
\hline IV & 1.791 & $1.271-2.522$ & $0.000^{* * *}$ \\
\hline \multicolumn{4}{|l|}{ Size } \\
\hline$<2$ & Reference & & \\
\hline $2-4$ & 1.314 & $1.096-1.577$ & $0.003^{* *}$ \\
\hline$\geq 4$ & 1.831 & $1.533-2.187$ & $0.000^{* * *}$ \\
\hline \multicolumn{4}{|l|}{ AJCC stage } \\
\hline । & Reference & & \\
\hline$\|$ & 1.609 & $1.311-1.975$ & $0.000^{* * *}$ \\
\hline III & 2.881 & $2.433-3.411$ & $0.000^{* * *}$ \\
\hline IV & 8.674 & $7.278-10.338$ & $0.000^{* * *}$ \\
\hline \multicolumn{4}{|l|}{ Surgery } \\
\hline Yes & Reference & & \\
\hline NO/Unknown & 2.936 & $2.481-3.475$ & $0.000^{* * *}$ \\
\hline \multicolumn{4}{|l|}{ Radiotherapy } \\
\hline Yes & Reference & & \\
\hline NO/Unknown & 0.715 & $0.608-0.841$ & $0.000^{* * *}$ \\
\hline \multicolumn{4}{|l|}{ Chemotherapy } \\
\hline Yes & Reference & & \\
\hline NO/Unknown & 1.254 & $1.112-1.413$ & $0.000^{* * *}$ \\
\hline
\end{tabular}

Notes: $* P<0.05 ; * * P<0.01 ; * * * P<0.001$

Abbreviations: ${ }^{\mathrm{a}} \mathrm{HR}$ hazard ratio, ${ }^{\mathrm{b}} \mathrm{Cl}$ confidence interval

respectively, for the nomogram, which were all higher than those for the AJCC staging system (0.791, 0.783, and 0.767$)$; the corresponding values for the validation cohort were $0.826,0.816,0.790,0.784,0.774$, and 0.755 , respectively (Fig. 3 ).

The NRI values for the 3-, 5-, and 8-year CSS probabilities were 0.219 (95\% confidence interval $[\mathrm{CI}]=$ $0.171-0.297$ ), 0.247 (95\% CI $=0.180-0.332$ ), and 0.259 (95\% CI $=0.195-0.358)$, respectively, in the training cohort, and 0.259 (95\% CI $=0.125-0.359), 0.272(95 \%$ $\mathrm{CI}=0.152-0.389)$, and $0.265(95 \% \mathrm{CI}=0.148-0.393)$ in the validation cohort. In addition, the IDI values for the 3-, 5-, and 8-year CSS probabilities were 0.029, 0.028, and 0.026 , respectively $(p<0.001)$, in the training cohort, $0.031,0.026$, and 0.025 , respectively $(p<0.001)$, in the validation cohort. All of the NRI and IDI values being greater than zero indicate that the new model had better discrimination ability than the AJCC staging system.

The calibration plots showed that the standard curves of the 3-, 5-, and 8-year CSS probabilities of the model was very close to the standard 45-degree diagonal lines and that the calibration points were evenly distributed, which demonstrated that the new model had good calibration ability (Fig. 4).

The abscissa in DCA curves is the threshold probability and the ordinate is the net benefit after the benefit is subtracted from the disadvantage [22]. Compared with the AJCC staging system, the 3-, 5-, and 8-year DCA curves were found to be enhanced for both the training and validation cohorts (Fig. 5).

\section{Discussion}

Previous studies of UTUC have been inadequate, with many clinical studies combining UTUC with renal or bladder cancer, which is not consistent with UTUC have its own unique pathological features [23]. The recent increases in the incidence and mortality rates of UTUC mean that the importance of determining the clinical prognosis of UTUC is also increasing [24]. The prognosis of UTUC is poor, and there is a lack of comprehensive and simple support research for this disease.

The special clinical characteristics of UTUC make it necessary to develop a UTUC-specific nomogram for providing more-accurate prediction models for use by clinical staff. In this study we successfully constructed a prognostic nomogram for UTUC patients using case data obtained from the SEER database. Nomograms are widely used in oncology and medicine to predict prognoses and meet the needs of clinical staff to provide patients with individualized treatments, and they are easier to understand than the traditional AJCC staging system [25]. The multivariable Cox regression analysis performed in the present study revealed that age at diagnosis, sex, race, marital status, histological grade, tumor size, AJCC stage, surgery status, radiotherapy status, and chemotherapy status are associated with the prognosis of UTUC. 


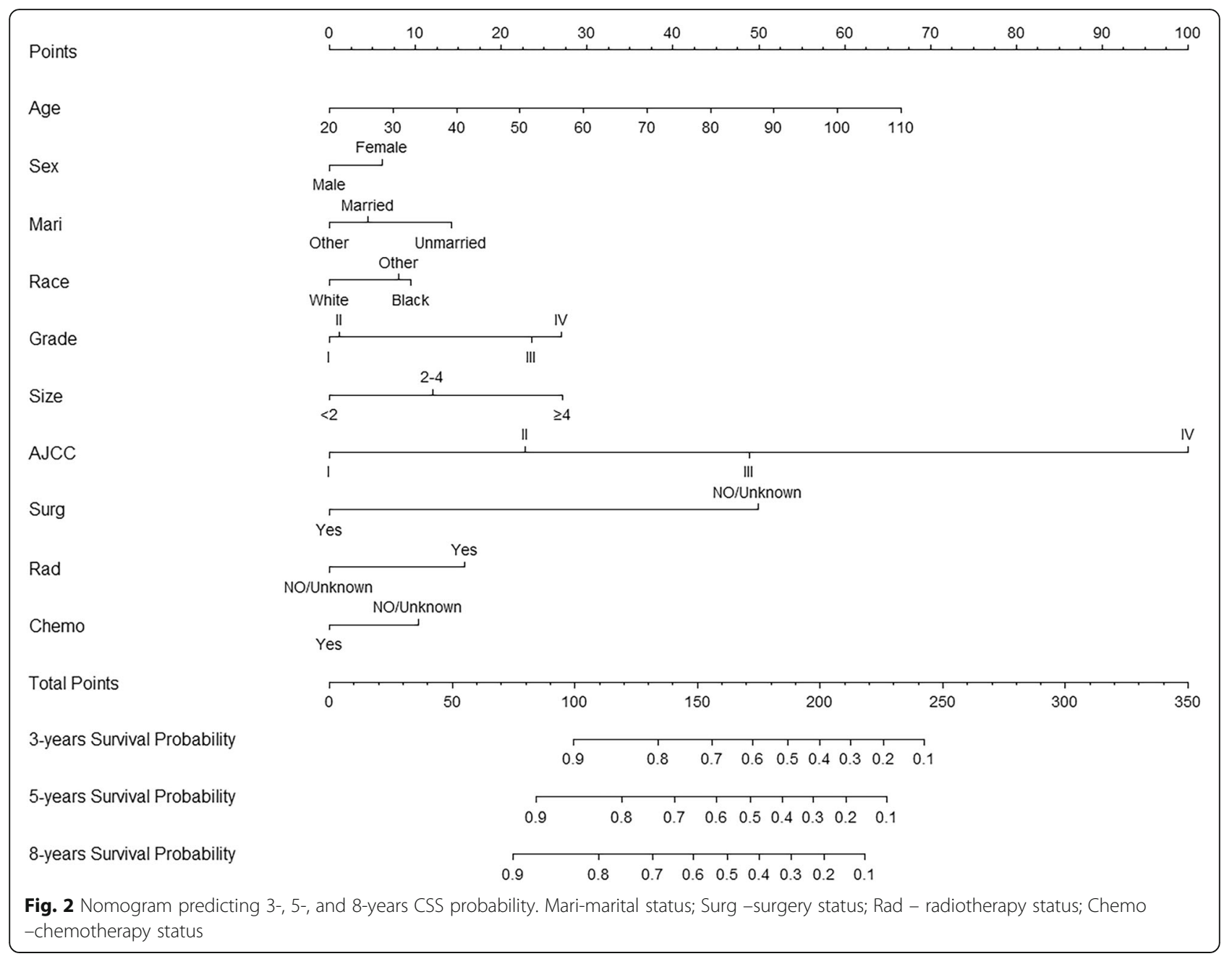

One of the prognostic factors included in the new model, age, has long been considered a risk factor for UTUC [26]. In contrast, whether sex is a risk factor for UTUC had not been determined previously [27], but the present study found that being female is a risk factor for survival $(\mathrm{HR}=1.142, p<0.01)$. Moreover, our study found for the first time that being unmarried is a risk factor of CSS affecting the prognosis of UTUC. A study showed that unmarried patients are at significantly higher risk of presentation with metastatic cancer, undertreatment, and death resulting from their cancer [28]. The relationship between marriage and cancer prognosis may be due to the following reasons. First of all, married patients may be higher than unmarried in terms of economic level and education level. Married persons also have better adherence to treatment, which may lead to differences in the prognosis of different marital status [29]. Second, a study showed that married patients were less likely to present with metastatic disease than those who were unmarried [28]. Finally, a review confirmed that marriage positively influences the likelihood of early diagnosis for all types of cancer. Correspondingly, if an unmarried person is diagnosed with cancer, the risk of developing advanced disease is greater, and the life expectancy is usually shorter [30]. In short, the prognosis of unmarried patients in this study is poor, and more reminders should be given to unmarried patients in this regard.

Histological grade, AJCC stage, surgery status, radiotherapy status, and chemotherapy status were also found to affect the survival probability. However, it is worth noting that the survival probability decreased in UTUC patients who received radiotherapy, which is consistent with the findings of Leow et al. [7] However, it should be noted that the gold standard treatment for UTUC is still surgery. Radiation therapy is usually performed in patients who have progressed to the point where surgery cannot be performed [31]. The experimental research on radiotherapy alone is very limited, which is worthy of further research in UTUC. On the other hand, this is a retrospective study and there are selection biases that are difficult to adjust. Therefore, the exact relationship 

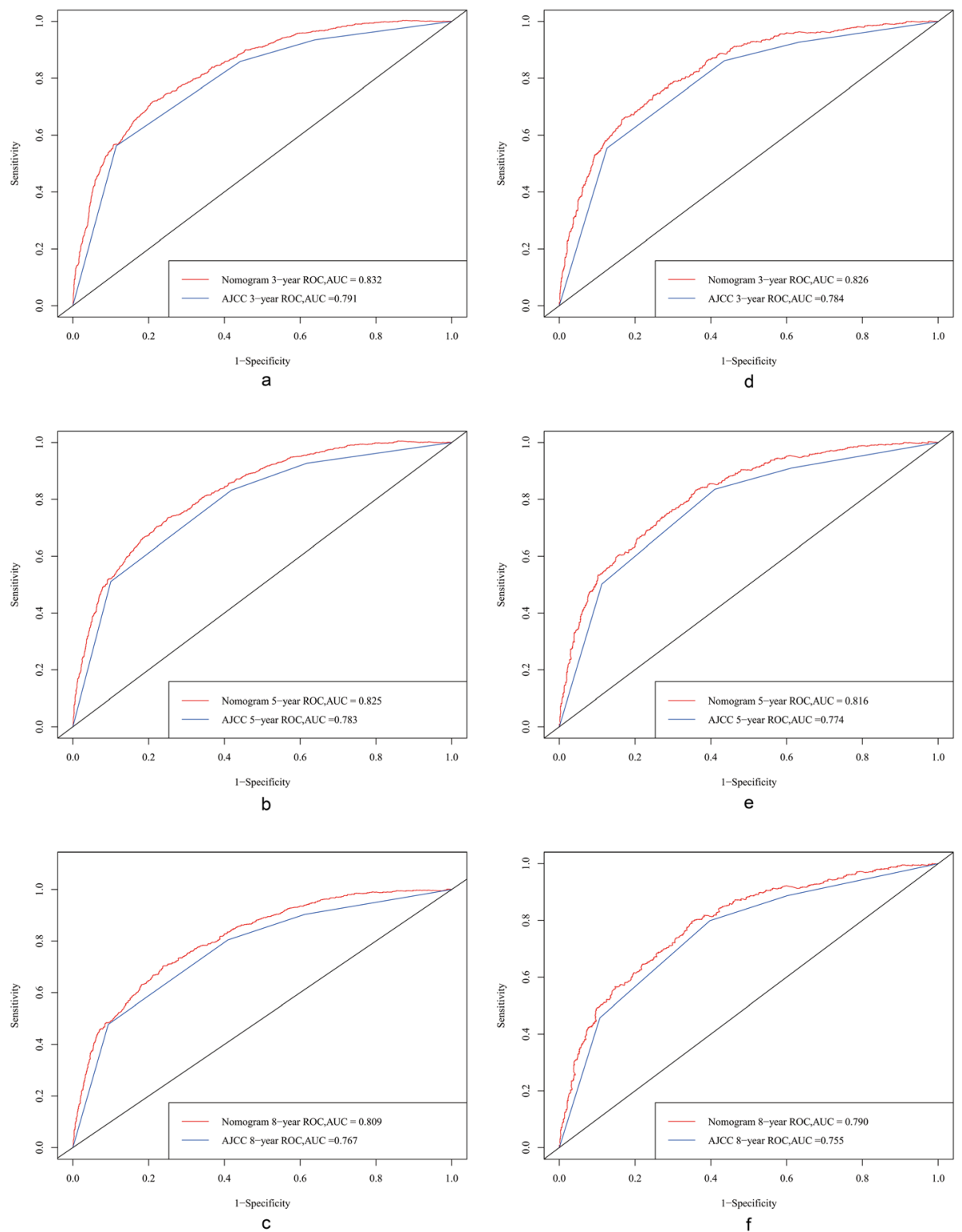

Fig. 3 ROC curves. The area under the ROC curve (AUC) was used to evaluate the performance of the new nomogram. $\mathbf{a}$, $\mathbf{b}$, and $\mathbf{c}$ represent the result of the training cohort; $\mathbf{d}$, $\mathbf{e}$, and $\mathbf{f}$ represent the result of the validation cohort

between radiotherapy and UTUC prognosis needs further prospective experiments to confirm. In addition, like some previous studies [32,33], tumor size was included in our model as a risk factor. However, the tumor site was not included in the model, meaning that this does not affect the prognosis of UTUC. Figure 2 clearly shows the relevant factors and their effects on the 3-, 5-, and 8-year CSS probabilities in UTUC patients. The total score can be obtained by adding the individual scores for each of the above factors, and clinical staff can use this score to predict the CSS probability of individual patients and thereby make decisions that are more likely to improve their prognosis.
After constructing the nomogram and analyzing related prognostic factors, it was compared with traditional the AJCC model using a training cohort and an internal validation cohort in order to evaluate the model underlying the nomogram. We used the $\mathrm{C}$-index and AUC to evaluate the discrimination performance, and found that both of these parameters were higher for the nomogram than for the AJCC staging system in both the training and validation cohorts (Fig. 3). When adding a new parameter to a model and then performing a comparison to see whether the predictive power of the model has improved, the increase in the AUC is sometimes not obvious. Instead, the NRI is often used to 

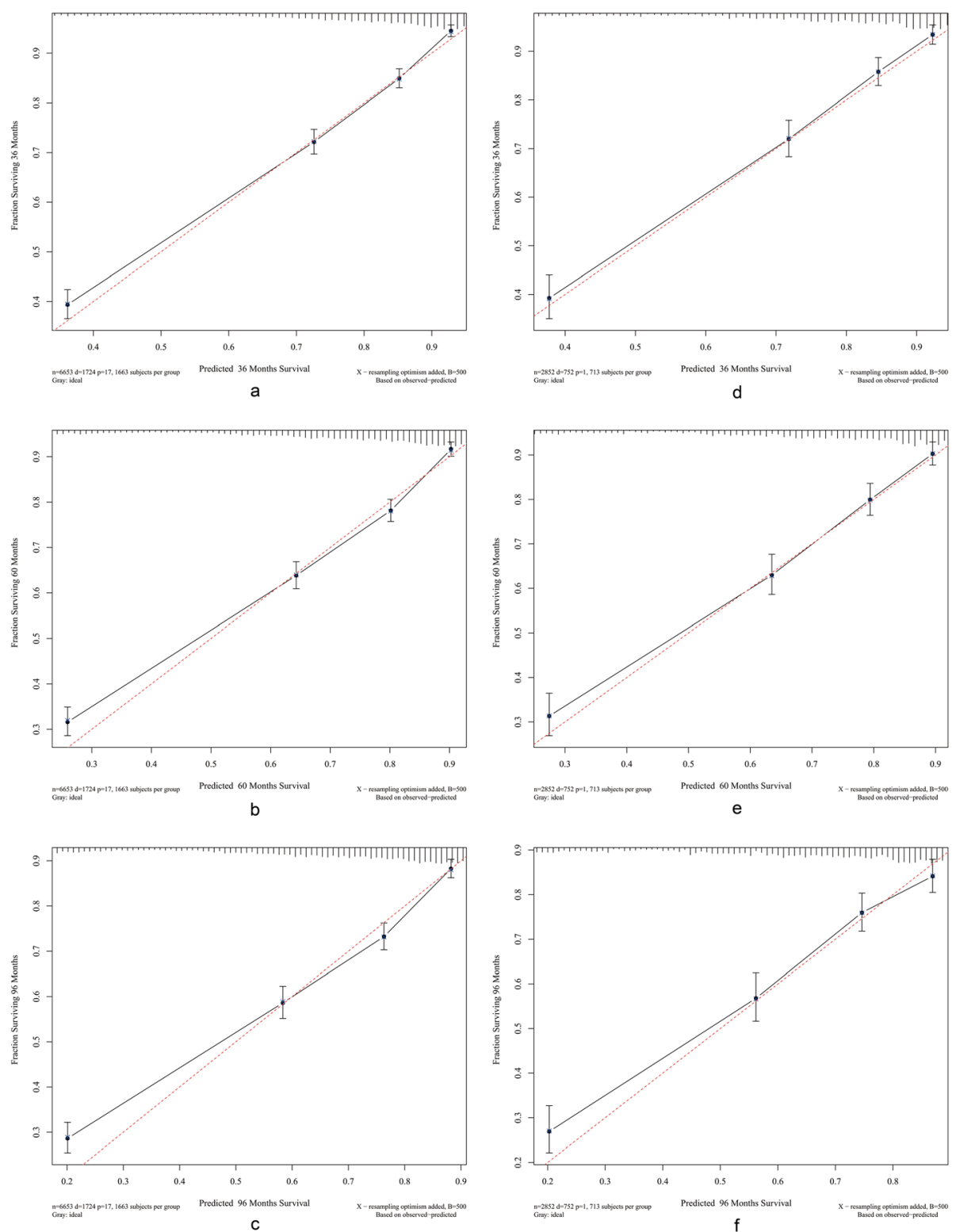

Fig. 4 Calibration curves. Calibration curves for 3-, 5-, and 8-years cancer specific survival probability depict the calibration of each model in terms of the agreement between the predicted probabilities and observed outcomes of the training cohort $(\mathbf{a}, \mathbf{b}, \mathbf{c})$ and validation cohort (d, e, $\mathbf{f})$

compare the prediction powers of two models, while the IDI can be used to reflect the overall model improvement $[34,35]$. The NRI of the prediction model showed that after adding the new index, the proportion of correct classifications for the 3-, 5-, and 8-year survival probabilities increased by $21.9,24.7$, and $25.9 \%$, respectively, in the training cohort, and by $25.9,27.2$, and $26.5 \%$ in the validation cohort $(p<0.001)$. The IDI revealed that the new model improved the predictive abilities for the 3-, 5-, and 8-year survival probabilities compared with the old model by $2.9,2.8$, and $2.6 \%$, respectively, in the training cohort, and by $3.1,2.6$, and $2.5 \%$ in the validation cohort $(p<0.001)$.
We used calibration curves to evaluate the calibration performance of the model. The 45-degree line in Fig. 4 is the standard line [36]. The broken lines in the figure are very close to the standard line and the predicted points are evenly distributed, which indicates that the nomogram exhibited good discrimination and calibration abilities both in the training and validation cohorts.

DCA is a method to evaluate prediction models by calculating the clinical net benefit. Figure 5 shows that the DCA curves of the nomogram for the 3-, 5-, and 8-year survival probabilities were almost all above those for the traditional AJCC model, which means that the new model has better clinical effectiveness. 

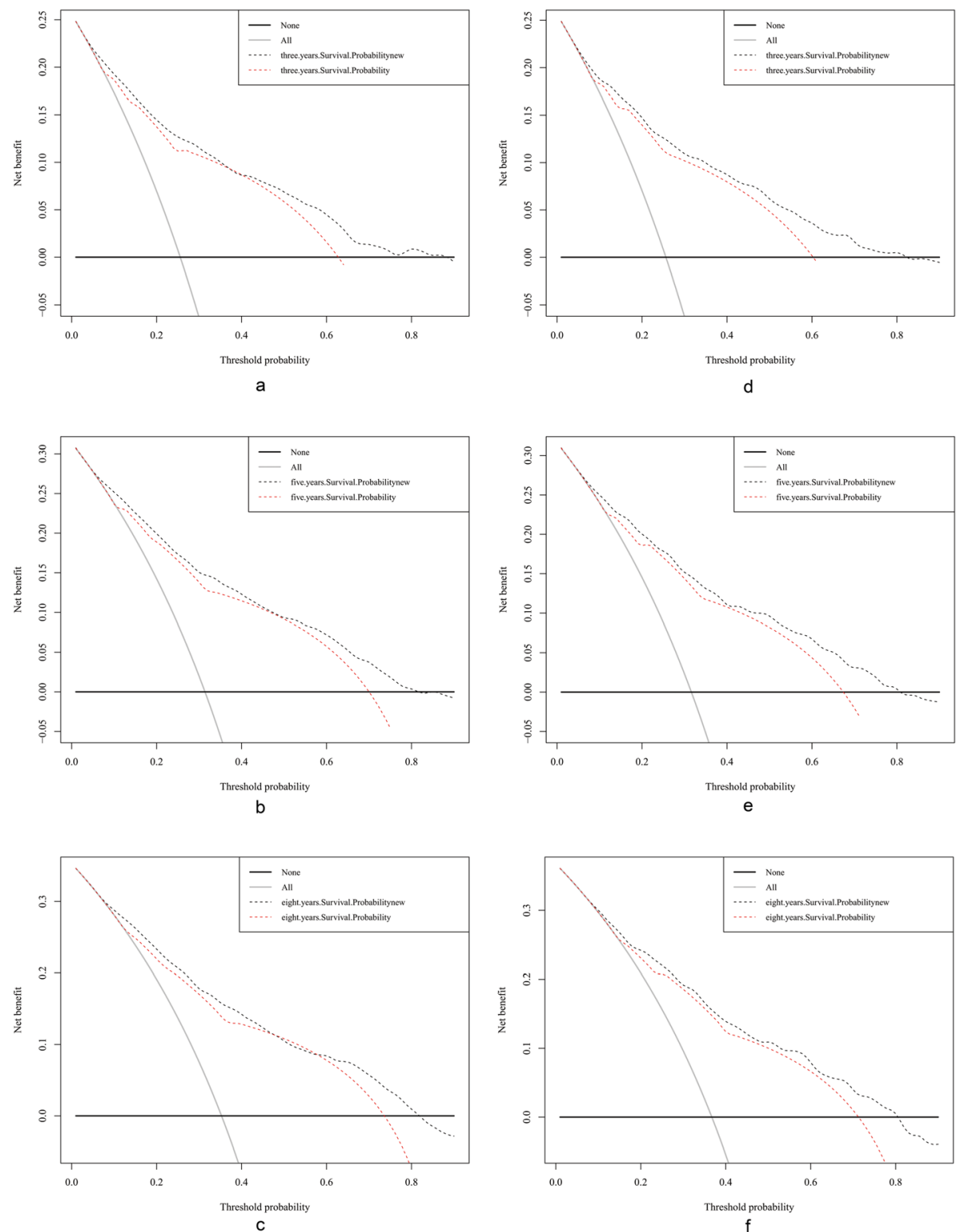

Fig. 5 Decision curve analysis curves. Decision curve analysis of the training cohort $(\mathbf{a}, \mathbf{b}, \mathbf{c})$ and validation cohort (d, e, $\mathbf{f})$ for 3-, 5-, and 8-years cancer specific survival probability

This study was subject to several limitations. The first limitation is that the study had a retrospective design and obtained data from the SEER database, which inevitably resulted in the presence of selection bias and information bias; for example, it is improper to integrate "no" and "unknown" into one category in the SEER database. The second limitation was that some potentially important factors were not included in the study, making it insufficiently comprehensive, such as certain biological indicators and behavioral habits. Finally, external validation of the nomogram was not performed, and the use of only internal validation may lead to overfitting of the new model. In the future we plan to incorporate more predictors and validate the effect of the model with external cohorts in order to obtain more-accurate results.

\section{Conclusion}

This study is the first to establish a comprehensive UTUC nomogram based on the SEER database and evaluate it using a series of indicators. A particularly interesting finding was of the marital status being a prognostic factor for UTUC. The tumor size also significantly affected the prognosis, will the primary site of UTUC did not. Our novel nomogram can be used a tool to help clinical staff to predict the 3-, 5-, and 8-year CSS 


\section{probabilities of UTUC patients more accurately than using the AJCC staging system.}

\section{Abbreviations}

UTUC: Upper-tract urothelial carcinoma; AJCC: American Joint Committee on Cancer; SEER: Surveillance, Epidemiology, and End Results; Cindex: Concordance index; AUC: Area under the time-dependent receiver operating characteristic curve; NRI: Net reclassification improvement; IDI: Integrated discrimination improvement; DCA: Decision curve analysis

\section{Acknowledgements}

Not applicable.

\section{Authors' contributions}

$J \mathrm{~L}$ and $\mathrm{CZL}$ designed the study; JY, FSX, and SZ collected and analyzed the data; CZL drafted the initial manuscript; $J L$ revised the article critically; KRE and DDH reviewed and edited the article; CZL and JY are co-first authors; JL and SPW are the correspondence authors. All authors approved the final manuscript.

\section{Funding}

The study was supported by The National Social Science Foundation of China (grant no. 16BGL183). The funding source played no role in research design and collection, analysis, report writing, and the decision to submit articles for publication.

\section{Availability of data and materials}

SEER collects cancer incidence data from population-based cancer registries covering approximately $34.6 \%$ of the U.S. population. SEER releases a standard set of research data every spring based on the previous November's submission of data from the registries. The data we used is based on the November 2018 submission. We accessed these through the SEER*Stat software with additional approvals. The data that support the findings of this study are available from the SEER*Stat software but restrictions apply to the availability of these data, which were used under license for the current study, and so are not publicly available. Data are however available upon reasonable request and with the SEER Research Data Agreement.

\section{Ethics approval and consent to participate}

Since cancer is a reportable disease in every state in the USA, informed patient consent is not required. When data usege agreement was signed, data on cancer research become available to the public.

\section{Consent for publication}

Not applicable.

\section{Competing interests}

The authors declare that they have no competing interests.

\section{Author details}

'Department of Clinical Research, The First Affiliated Hospital of Jinan University, Guangzhou, Guangdong, China. ${ }^{2}$ School of Public Health, Xi'an Jiaotong University Health Science Center, Xi'an, Shaanxi, China. ${ }^{3}$ School of Public Health, Shaanxi University of Chinese Medicine, Xi'an, Shaanxi, China. ${ }^{4}$ Cardiovascular Research Center, School of Basic Medical Sciences, Xi'an Jiaotong University Health Science Center, Xi'an 710061, People's Republic of China. ${ }^{5}$ Key Laboratory of Environment and Genes Related to Diseases of Ministry of Education, Xi'an Jiaotong University Health Science Center, Xi'an, Shaanxi, China.

\section{Received: 17 December 2019 Accepted: 28 May 2020} Published online: 08 June 2020

\section{References}

1. Roupret M, Babjuk M, Comperat E, et al. European Association of Urology guidelines on upper urinary tract Urothelial carcinoma: 2017 update. Eur Urol. 2018;73(1):111-22.

2. Raman JD, Messer J, Sielatycki JA, Hollenbeak CS. Incidence and survival of patients with carcinoma of the ureter and renal pelvis in the USA, 19732005. BJU Int. 2011;107(7):1059-64.
3. Siegel RL, Miller KD, Jemal A. Cancer statistics, 2019. CA Cancer J Clin. 2019; 69(1):7-34.

4. Siegel RL, Miller KD, Jemal A. Cancer statistics, 2018. CA Cancer J Clin. 2018; 68(1):7-30.

5. Siegel RL, Miller KD, Jemal A. Cancer statistics, 2017. CA Cancer J Clin. 2017; 67(1):7-30.

6. Seaman EK, Slawin KM, Benson MC. Treatment options for upper tract transitional-cell carcinoma. Urol Clin North Am. 1993;20(2):349-54

7. Leow JJ, Martin-Doyle W, Fay AP, Choueiri TK, Chang SL, Bellmunt J. A systematic review and meta-analysis of adjuvant and Neoadjuvant chemotherapy for upper tract Urothelial carcinoma. Eur Urol. 2014;66(3): 529-41.

8. Krogh J, Kvist E, Rye B. Transitional cell carcinoma of the upper urinary tract: prognostic variables and post-operative recurrences. Br J Urol. 1991;67(1): 32-6.

9. Amin MB, Greene FL, Edge SB, et al. The Eighth Edition AJCC Cancer Staging Manual: Continuing to build a bridge from a population-based to a more "personalized" approach to cancer staging. CA Cancer J Clin. 2017; 67(2):93-9.

10. Novara G, De Marco V, Gottardo F, et al. Independent predictors of cancerspecific survival in transitional cell carcinoma of the upper urinary tract: multi-institutional dataset from 3 European centers. Cancer. 2007;110(8): 1715-22.

11. Cha EK, Shariat SF, Kormaksson M, et al. Predicting clinical outcomes after radical Nephroureterectomy for upper tract Urothelial carcinoma. Eur Urol. 2012;61(4):818-25.

12. Xylinas E, Kluth L, Passoni N, et al. Prediction of intravesical recurrence after radical nephroureterectomy: development of a clinical decision-making tool. Eur Urol. 2014;65(3):650-8.

13. lasonos A, Schrag D, Raj GV, Panageas KS. How to build and interpret a Nomogram for Cancer prognosis. J Clin Oncol. 2008;26(8):1364-70.

14. Surveillance Research Program, National Cancer Institute SEER*Stat software (seer.cancer.gov/seerstat) version $\langle$ SEER*Stat 8.3.6 .

15. Surveillance, Epidemiology, and End Results (SEER) Program (www.seer. cancer.gov) Research Data (1975-2016), National Cancer Institute, DCCPS, Surveillance Research Program, released April 2019, based on the November 2018 submission.

16. Yang J, Liu QQ, Geng H, Tian GX, Zeng XT, Lyu J. SEER database application and data extraction methods and processes. Chin J Evid Based Cardiovasc Med. 2018;10(07):781-4.

17. Simone G, Papalia R, Loreto A, Leonardo C, Sentinelli S, Gallucci M. Independent prognostic value of tumour diameter and tumour necrosis in upper urinary tract urothelial carcinoma. BJU Int. 2009; 103(8):1052-7.

18. Hilden J, Gerds TA. A note on the evaluation of novel biomarkers: do not rely on integrated discrimination improvement and net reclassification index. Stat Med. 2014:33(19):3405-14.

19. Parikh CR, Coca SG, Thiessen-Philbrook H, et al. Postoperative biomarkers predict acute kidney injury and poor outcomes after adult cardiac surgery. J Am Soc Nephrol. 2011;22(9):1748-57.

20. Callegaro D, Miceli R, Bonvalot $\mathrm{S}$, et al. Development and external validation of two nomograms to predict overall survival and occurrence of distant metastases in adults after surgical resection of localised softtissue sarcomas of the extremities: a retrospective analysis. Lancet Oncol. 2016;17(5):671-80

21. Vickers AJ, Elkin EB. Decision curve analysis: a novel method for evaluating prediction models. Med Decis Mak. 2006;26(6):565-74.

22. Stewart GD, Bariol SV, Grigor KM, Tolley DA, McNeill SA. A comparison of the pathology of transitional cell carcinoma of the bladder and upper urinary tract. BJU Int. 2005;95(6):791-3.

23. Balachandran VP, Gonen M, Smith JJ, DeMatteo RP. Nomograms in oncology: more than meets the eye. Lancet Oncol. 2015;16(4):e173-80.

24. Eylert MF, Hounsome L, Verne J, Bahl A, Jefferies ER, Persad RA. Prognosis is deteriorating for upper tract urothelial cancer: data for England 1985-2010. BJU Int. 2013;112(2):e107-13.

25. Lughezzani $G$, Jeldres $C$, Isbarn $H$, et al. Nephroureterectomy and segmental ureterectomy in the treatment of invasive upper tract urothelial carcinoma: a population-based study of 2299 patients. Eur J Cancer. 2009:45(18):3291-7.

26. Vickers AJ, van Calster B, Steyerberg EW. A simple, step-by-step guide to interpreting decision curve analysis. Diagn Progn Res. 2019;3:18. 
27. Fernandez MI, Shariat SF, Margulis V, et al. Evidence-based sex-related outcomes after radical nephroureterectomy for upper tract urothelial carcinoma: results of large multicenter study. Urology. 2009;73(1):142-6.

28. Aizer AA, Chen M-H, McCarthy EP, Mendu ML, Koo S, Wilhite TJ, Graham PL, Choueiri TK, Hoffman KE, Martin NE, et al. Marital status and survival in patients with cancer. J Clin Oncol. 2013;31(31):3869-76.

29. Cohen SD, Sharma T, Acquaviva K, Peterson RA, Patel SS, Kimmel PL. Social support and chronic kidney disease: an update. Adv Chronic Kidney Dis. 2007;14(4):335-44.

30. Buja A, Lago L, Lago S, Vinelli A, Zanardo C, Baldo V. Marital status and stage of cancer at diagnosis: A systematic review. Eur J Cancer Care. 2018; 27(1):e12755.

31. Leow JJ, Orsola A, Chang SL, Bellmunt J. A contemporary review of management and prognostic factors of upper tract urothelial carcinoma. Cancer Treat Rev. 2015:41(4):310-9.

32. Margulis V, Shariat SF, Matin SF, et al. Outcomes of radical nephroureterectomy: a series from the upper tract Urothelial carcinoma collaboration. Cancer. 2009;115(6):1224-33.

33. Raman JD, Ng CK, Scherr DS, et al. Impact of tumor location on prognosis for patients with upper tract urothelial carcinoma managed by radical nephroureterectomy. Eur Urol. 2010;57(6):1072-9.

34. Iki M, Fujita Y, Tamaki J, et al. Trabecular bone score may improve FRAX(R) prediction accuracy for major osteoporotic fractures in elderly Japanese men: the Fujiwara-kyo osteoporosis risk in men (FORMEN) cohort study. Osteoporos Int. 2015;26(6):1841-8.

35. Chambless LE, Cummiskey CP, Cui G. Several methods to assess improvement in risk prediction models: extension to survival analysis. Stat Med. 2011;30(1):22-38.

36. Steyerberg EW, Vickers AJ, Cook NR, et al. Assessing the performance of prediction models: a framework for traditional and novel measures. Epidemiology. 2010;21(1):128-38.

\section{Publisher's Note}

Springer Nature remains neutral with regard to jurisdictional claims in published maps and institutional affiliations.

Ready to submit your research? Choose BMC and benefit from:

- fast, convenient online submission

- thorough peer review by experienced researchers in your field

- rapid publication on acceptance

- support for research data, including large and complex data types

- gold Open Access which fosters wider collaboration and increased citations

- maximum visibility for your research: over $100 \mathrm{M}$ website views per year

At $\mathrm{BMC}$, research is always in progress.

Learn more biomedcentral.com/submissions 